\title{
GESNERUS
}

Vierteljahrsschrift für Geschichte der Medizin und der Naturwissenschaften Revue trimestrielle d'histoire de la médecine

Jahrgang/Vol. 24 1967 Heft/Fasc. $1 / 2$

\section{Autobiographisches von Johann Jakob Wepfer (1620-1695) in einem Briefwechsel mit Johann Conrad Brotbeck (1620-1677)}

\author{
Von P.Eichenberger
}

Der Anlaß zu dieser Veröffentlichung gab eine achtseitige Kopie eines Briefes von Wepfer an Brotbeck. Sie ist von Wepfers eigener Hand geschrieben. Dieser Brief enthält neben damaligen Problemen des Blutkreislaufes auch noch wertvolle bis jetzt unveröffentlichte, autobiographische Angaben Wepfers über seine Studienzeit und gibt auch einen Einblick in die damaligen Streitschriften um den Blutkreislauf. Wepfers Brief bildet den Kern dieser Arbeit, die als vorläufige Publikation gedacht ist und der daher auch der Mangel der teilweisen Unvollständigkeit anhaftet. Auch konnten noch nicht alle wünschenswerten Unterlagen gefunden werden, so z. B. der handschriftliche Nachlaß von Brotbeck.

\section{Das Material}

Eine Fußnote von H.Fischer in seiner Veröffentlichung des Briefwechsels zwischen Johann Jakob Wepfer und seinem Sohn Johann Conrad ${ }^{1}$ führte 1962 zur Wiederentdeckung von J.J. Wepfers handschriftlichem Nachlaß. Er wurde 1774, nach dem Tode des letzten direkten Nachkommen

1 H.Fischer, Briefe Johann Jakob Wepfers (1620-1695) an seinen Sohn Johann Conrad (1657-1711), Studiosus medicinae zu Basel und Leyden, Aarau/Leipzig 1943, S. 5, Anm. 1: «Weiteres handschriftliches Material findet sich auf der Universitätsbibliothek in Leyden .... 
- Georg Michael Wepfer (1692-1774) -, nebst der Bibliothek von ungefähr 1600 Bänden, von der Medizinischen Fakultät in Leiden (Holland) für 600 Gulden (120 Golddukaten) erworben. Dieser Nachlaß umfaßt rund 12000, meist lateinisch beschriebene Blätter in Quartformat, und ist in 46 Faszikel eingeteilt ${ }^{2}$. Ein Faszikel aus diesem Nachlaß, 122 Blätter umfassend, wurde von Wepfer selbst mit «Consilia et Epistolae» betitelt und liegt in gebundener Form vor ${ }^{3}$. Darin findet sich u.a. aus Wepfers eigener Hand eine achtseitige Kopie seines Briefes an den Tübinger Stadtarzt JohanN Conrad Brotbeck (1620-1677), datiert vom 1. September 1656. Dem Inhalt konnte entnommen werden, daß ein Brief Brotbecks an Wepfer vorausgegangen sein mußte. Dieses Schreiben konnte dann im Wepfer-Nachlaß der Zentralbibliothek Zürich aufgefunden werden ${ }^{4}$. Es ist vom 26. Juni 1656 datiert. Unter der gleichen Signatur findet sich ein weiterer Brief Brotbecks. Im Inhalt schließt er unmittelbar an den vorherigen an und ist vom Jahresende 1658 datiert. Fünf weitere Brotbeck-Briefe aus den Jahren 1673-1675 sind in der Universitätsbibliothek in Leiden ${ }^{5}$. Sie wurden hier nicht berücksichtigt.

\section{Die Korrespondenten}

JohanN JaKob WePfer (1620-1695) wurde am 23.Dezember 1620 als der älteste Sohn des Zunftmeisters, Richters und Ratsherrs Georg MichaEL WePFER (1591-1659) in Schaffhausen geboren. Am Collegium Humanitatis seiner Vaterstadt erhielt er seine erste Ausbildung. Schon mit 18 Jahren immatrikulierte er sich im April 1638 an der Universität in Basel. Jedoch schon im nächsten Jahre wechselte er an die Universität Straßburg (siehe Brief), wo er bis 1643 blieb, aber noch im selben Jahre nach Basel zurückkehrte. 1644 studierte Wepfer in Padua, und 1645 finden wir ihn in Venedig ${ }^{6}$, 1646 in Rom $^{7} .1647$ promovierte er in Basel zum Doktor der Medizin ${ }^{8}$ und

2 Eine Auswertung dieses Materials wird als Dissertation (P. Eichengerger, Johann Jakob Wepfer (1620-1695) als klinischer Praktiker) in den Basler Veröffentlichungen zur Geschichte der Medizin und der Naturwissenschaften» 1967 erscheinen.

${ }^{3}$ Leiden Universitätsbibliothek (LUB) BPL 249, fasc. 12, fol. 1-122.

${ }^{4}$ Zentralbibliothek Zürich (ZBZ), Ms Z VII 295.7, S. 1-4 und S. 5-8.

5 LUB BPL 249, literae A-Z.

${ }^{6}$ LUB BPL 249, fasc. 12, fol. 106 R.

7 Auch in J. J. WePFER, Observationes medico-practicae de affectibus capitis internis et externis, Schaff hausen 1727 (posthum), S. 40.

8 J.J.WEPFER, Dissertatio inauguralis de palpatione cordis, Basileae 1647. 
wurde noch im gleichen Jahre zum Stadtarzt in Schaffhausen gewählt. In dieser Eigenschaft wurde er bald Nachfolger in verschiedenen Ämtern, so 1650 Klosterarzt von Rheinau nach dem Tode von Johann Screta (gestorben 7.Januar 1650) ${ }^{9}$. Im gleichen Jahre vermählte er sich mit BARBARA Rink von Wildenberg. 1658 veröffentlichte er sein erstes und bedeutendes Werk über die Apoplexie ${ }^{10} .1675$ wurde er Leibarzt der Herzöge von Württemberg und des Markgrafen Friedrich von Baden-Durlach. 1685 wurde er noch als Leibarzt des Kurfürsten Karl von der Pfalz berufen. Wepfer war der führende Kopf der damaligen freien Ärzteschule in Schaffhausen. Zu seinen Mitarbeitern zählte sein Schwiegersohn Johann Conrad Brunner (1653-1727), Johann Conrad Peyer (1653-1711) und Heinrich Screta (1637-1689), um nur die bedeutendsten zu nennen.

Johann Conrad Brotbeck (1620-1677) 11 wurde am 28.(29.) August 1620 in Tübingen geboren und starb dort auch am 22. Februar 1677. Er wurde am 18.0ktober 1633 in der Artistenfakultät des Universität Tübingen immatrikuliert, erwarb das Baccalaureat am 31.August 1636 und ließ sich nach dem Studium der Eloquenz, Philosphie, Mathematik und Physik am 15. Januar 1641 in die medizinische Fakultät eintragen und promovierte am 9. August 1646 zum Doktor der Medizin. Einen Tag später verheiratete er sich mit Christine Bardili (1600-1647). 1646 bis 1650 war er Stadtphysikus in Eßlingen, 1650 wurde er a.o. Professor für Astronomie in Tübingen, 1653 o. Professor der Physik, 1657 o. ö. Professor der Medizin. Er war sechzehnmal Dekan der Medizinischen Fakultät, zweimal (1662 und 1668/69) Rektor der Universität Tübingen. Von 1655 bis 1676 erschienen von ihm veranlaßt oder von ihm verfaßt ungefähr 40 Dissertationen mit physikalischen und medizinischen Themen.

\section{Der Briefwechsel}

Beide Korrespondenten standen in ihrem 36. Lebensjahr, als dieser Briefwechsel begann. Wepfer scheint keine akademischen Ambitionen gehabt zu

9 LUB BPL 249 , fasc. 4 , fol. 39 R.

10 J.J. WePFER, Observationes anatomicae ex cadaveribus eorum, quos sustulit apoplexia, Schaff hausen 1658.

11 Diese Mitteilung erhielt ich von Herrn Dr. R.Toellner, Institut für Geschichte der Medizin der Universität Tübingen. Ihm und Herrn Prof. Dr. W.von Brunv sei an dieser Stelle für diese Auskunft recht herzlich gedankt. 
haben, während Brotbeck bereits Professor für Astronomie und Physik an der Universität Tübingen war. Brotbeck hat als erster den Briefwechsel eröffnet, und zwar über einen gemeinsamen Freund, den Chirurgen JoHanN Christoph Simon ${ }^{12}$. Dieser muß Brotbeck Bemerkenswertes über Wepfer erzählt haben, am wahrscheinlichsten sind Berichte über Sektionen, die Wepfer ausgeführt hatte. Wepfer hatte nämlich kurz nach seiner Berufung zum Stadtarzt 1647 sich von den Behörden der Stadt die Sektionserlaubnis geholt. Brotbeck scheint in dieser Hinsicht weniger glücklich gewesen zu sein und suchte nun Bereicherung bei Wepfer. Die Adresse von Brotbecks erstem Brief lautet:

\author{
Nobili et Excellentissimo \\ Viro, Dno. Jo: Jacobo Wö- \\ pfero, utr. Med. Doct. Eximio \\ et poliatro Scaphusiano \\ Experimentissimo ac fe- \\ licissimo, Fautori \\ suo percolendo
}

Schaffhausen.

Brotbeck schreibt in etwas schwülstigem und bilderreichem Stil folgendes an Wepfer ${ }^{13}$ :

Gratiam et amorem Jesu!

cum officiorum promptitudine!

Nobilis, Excellentissime et Experientissime Vir, Domine Fautor percolende.

Idem literatorum, quod mercatorum ordini frequens est, variis inter sese uti et exercere se commerciis. Chartae autem literariae, sunt naves mercatoria, quibus ad TE quoque transnavigare, libido incessit, scilicet ut reciprocas quoque Tuas ditissimis doctrinae mercibus instructas provocem et exorem. Vadum hoc aperuit Dominus Simonius, Affinis et amicus meus singularis, qui apud TE, meae mentio-

12 Johann Christoph Simon stammte aus Tübingen und war Kammerdiener und Hauschirurge von Franz Karl, des Grafen von Fürstenberg. Wepfer nennt Simon sehr erfahren und bewährt. Er erwähnt ihn in seinen Observationes ... de affectibus capitis an mehreren Stellen (so S. 8, 11, 12, 23, 34, 677, 682, 944). Wie Wepfer an diesen Stellen schreibt, kann man annehmen, daß er ihn besonders geschätzt haben muß.

13 ZBZ, Ms Z VII 295.7, S. 1 (Text) und S. 4 (Anschrift). 
nem fecit tenuitatis, recensens, quid circa sectionem hydropicae cuiusdam, a nobis visum, gestum, quidque me inter et alios varie de sanguinis circulatione, cordisque sanguificatione disputatum sit. Quam observationem notatu dignam, Excellentiae Tuae legendam communico. Quoad circulationem et sanguificinam, ut ita loquor, miror cur seniores plerunque medici, quos inter noster Dominus Doctor Hafenreffer et Dominus Doctor Gerhardus, quin et ille fuit medicorum ocellus, Clarissimus Casparus Hofmannus atque etiamnum hodie contra Thom. Bartholiunum Riolanus, miror, inquam, cur autopsiae repugnare ausint, metuentes fortasse, ne coelum nostrum Apollineum ruat. Ego jam ante hoc duodecennium illam laetsi oculis aspectavi ex hyperborea oceano emersam Venerem. Cuius quoque imaginem in Sacri codicis amphitheatro, emblematica sive hieroglyphice depictam suspensamque observavi, et solenni dissertatione, sicca transmissa, sed crassa Minerva, delineatam in actu meo doctoreo, decennio abhinc, asserere conatus fui. Unice nun efflagito, ut curtis hisce suppellectilibus meis, opulentas judicii, eruditionis, et autopsiae, quibus omnibus polles, anatomicae merces tuas adjicero, commercioque hoc literario, ni grave fuerit, identidem me locuplentare nitaris. Ignosce interea rudi et impudenter te adorienti calamo meo, cui tamen meus erga $\mathrm{TE}$ amor, spesque benevolentiae tuae concepta veniam dabit. Vale peregregium artis nostrae. Secus! et me, prout feliciter incepisti, ama. Dabam currente calamo, e Museo, Thubingae, die 26. Junii A.C. 1656

Excellentiae Tuae

Deditissimus

Remittenda peto inclusa

hac manuscripta

Joh. Conradus Brotbequius, MD

et phys. p. ordin.

«Vornehmer, ausgezeichneter und erfahrener Mann, sehr geehrter Herr und Gönner.

Auch die Wissenschafter verkehren mit Verschiedenen und pflegen Umgang mit ihnen, wie das beim kaufmännischen Stande üblich ist. Die wissenschaftlichen Briefe sind wie Handelsschiffe mit denen ich zu Dir übersetzen kann, wenn mich die Lust ankommt. Ich ersuch Dich jedoch auch um zurückfahrende, die mit Deinen, unser Fach bereichernden Gütern beladen sind. Diesen Zugang öffnete Herr Simon ${ }^{12}$, mein Schwager und bester Freund, der, als er bei Dir war, meine Dürftigkeit erwähnte und erzählte, was wir bei der Sektion einer wassersüchtigen Frau, die wir gesehen und obduziert hatten, feststellten und was von mir mit den andern über Blutkreislauf und Blutbildung verhandelt wurde. Diese der Niederschrift würdige Beobachtung sende ich Dir zum Lesen. Was den Blutkreislauf und die Blutbildung betrifft, um es so zu sagen, wundere ich mich, warum die oft älteren Ärzte - 
bei uns die Herren Doktores Hafenreffer ${ }^{14}$ und Gerhard ${ }^{15}$; der berühmte Caspar Hofmann ${ }^{16}$, der sogar ein Augapfel der Ärzte gewesen ist, und jetzt Riolan ${ }^{17}$ gegen Thomas Bartholin ${ }^{18}$-, ich wundere mich, wie ich sagte, warum diese die Autopsie zurückzuweisen wagen; vielleicht weil sie befürchten, daß unser schöner Himmel zusammenstürzt? Ich habe die Autopsie schon vor zwölf Jahren als eine aus dem nördlichen Meere entstandene Venus freudig betrachtet. Deren Bild aber habe ich im Amphitheater, stückweise oder unklar dargestellt und aufgehängt, beobachtet und in einer feierlichen Dissertation ${ }^{19}$ bei meiner Promotion vor zehn Jahren, schlicht, aber mit Verstand nachzuzeichnen versucht. Ich erstrebe nun das eine: Ich möchte meinem mangelhaften Grundstock Deine, an Urteil, Unterweisung und Autopsien reichen Verdienste hinzufügen, denn die besitzest Du ja. Wenn auch der heutige wissenschaftliche Bericht nicht gewichtig war, bereichere mich bitte des öftern. Verzeih inzwischen den mangel-

14 Samuel Hafenreffer (1587-1660) wurde in Herrenberg geboren, war Arzt und praktizierte in Kirchheim. Später wurde er noch Professor der Medizin in Tübingen.

15 Johann Gerhard war Doktor der Medizin und Professor der Medizin an der Universität in Tübingen. In scinen Schrifţen beschäftigte er sich hauptsächlich mit Alchemie.

16 Caspar Hofmann (1572-1648) wurde in Gotha in ärmlichen Verhältnissen geboren. Er studierte Medizin in Leipzig, Straßburg, Altorf und drei Jahre in Padua, schließlich schloß er seine Studien in Basel 1605 ab. Zuerst war er «Pestarzt» in Nürnberg und wurde 1608 Professor der Medizin in Altorf, wo er auch starb. Er war der Repräsentant steriler Buchgelehrtheit.

17 Jean Riolan der Jüngere (1580-1657) war Professor für Anatomie in Paris und wohl der heftigste Gegner Harveys und dessen Anhänger. Riolan brachte sehr begründete Argumente gegen eine kritiklose Annahme einer neuen Lehre vor (siehe Anm. 21).

18 Thomas Bartholin (1616-1680) wurde als dritter von sechs Söhnen geboren. Er studierte in Leiden drei Jahre lang Philosophie, Philologie, Arabisch, Theologie und Medizin. Anschließend reiste er durch Holland, Frankreich und Italien. Er soll insgesamt neun Jahre an den Universitäten von Leiden, Paris, Montpellier, Padua und Basel studiert haben. Er wurde 1642 in Padua Konsiliar und Protektor der Deutschen Nation. Seine Studien schloß er am 25. September 1645 in Basel ab. Bereits 1647 wurde er Professor für Mathematik in Kopenhagen und 1648 erhielt er die Professur für Anatomie. 1656 ernannte man ihn zum «Decanatus perpetuus Collegii medici». 1661 legte er sein Amt nieder und wurde Honorarprofessor. 1670 verbrannte sein Landhaus Hagestedgaard bei Kopenhagen und Bartholins ganze, reichhaltige Bibliothek. Er wurde gleichsam als Trost zum Königlichen Rat und Leibarzt ernannt. 1672 wurde er Bibliothekar der Akademie und starb am 4. Dezember 1680.

19 Johann Conrad Brotbeck (1620-1677), Dissertatio inauguralis, Titel unbekannt bisher, stattgefunden am 9. August 1646 in Tübingen. Mitteilung von Dr. R. Toellner, Institut für Geschichte der Universität Tübingen. 
haften und aufdringlichen Brief, der dennoch meine Zuneigung und die Hoffnung auf Deine gütige Aufnahme darlegen möchte. Lebe wohl, lieber Kollege, und bleibe mir, wie Du bereits begonnen hast, verbunden.

Tübingen, Bibliothek, 26. Juni 1656

Die beigelegten Manuskripte ${ }^{20}$

erbitte ich zurück.
Dein Dir sehr ergebener

Johann Conrad Brotbeck

Arzt und Stadtarzt. »

Brotbeck wirbt in diesem Brief um die Freundschaft mit Wepfer und erhofft durch Erfahrungsaustausch eine gegenseitige Bereicherung, vor allem auf dem Gebiet der Sektion. Im Zusammenhang damit kommt er auch auf das brennendste Problem der damaligen Zeit zu sprechen: William Harveys Blutkreislauf. Er spielt auf den damals ziemlich heftigen Kampf für oder gegen den Blutkreislauf an ${ }^{21}$.

Wepfers Originalbrief ist uns nicht erhalten, wohl aber eine eigenhändige Kopie seines Antwortschreibens. Wepfer sieht sich durch Brotbecks Brief soweit herausgefordert, daß er ganz eindeutig und klar Stellung für den Blutkreislauf bezieht. Schon nach gut zwei Monaten antwortet er folgen$\operatorname{des}^{22}$ :

d. 1. Sept. 1656 ad Joh. Conradium Brotbequium, MD et P. Physicum Tubingensem

Salve Nobilis Excellentissimime et Clarissime Domine Fautor et Amice plurimum colende.

Pridem mihi Excellentiae Tuae multigoga eruditio et singulare rem medicam illustrandi studium a Domino Simonio communi nostro amico innotuit. Pridem et Excellentiae Tuae amititiam (sic!) ambire mecum statui. Novin. quo quis magis literis imbutus est eo et humaniorem esse nec facile fastidire eos qui in eadem palestra, licet non pari fuerunt qui scintillam in pectore absconditam in

20 Diese Manuskripte sind wahrscheinlich in Brotbecks Nachlaß zu finden. Ein solcher ist, wie mir Herr Dr. R. Toellner vom medizingeschichtlichen Institut in Tübingen mitteilte, bezeugt, jedoch weiß man noch nicht, ob und wo er sich erhalten hat.

21 N.MANI hat sich im Gesnerus 18 (1961) 85-146, ausführlich über «Darmresorption und Blutbildung im Lichte der experimentellen Physiologie des 17. Jahrhunderts » geäußert. Darin sind die bedeutenden Werke und auch die Streitschriften um den Blutkreislauf erwähnt. - Neuerdings erschienen in den Documenta Geigy einige kleine Hefte über das gleiche Problem, verfaßt von Th. Vetter und übersetzt von F. Kudlien. Darin wird übersichtlich auf diese Streitschriften eingegangen.

22 LUB BPL 249, fasc. 12, fol. 92 R-95 V. 
flammam erumpere compullerunt. Utinam advoto et exspectationi tuae respondere possum! Quam sit mihi carta supellex haud ignoro, et si forte in uno alterove ad palatum scribere valerem, cum tunc ab Exercitiis Academicis diu jam abfuerim et ut Dominus Simonius novit quotidie practicis occupationibus ab his studiis avellar, vel in una alterove accuratus ad lubitum Excellentiae Tuae et Academicam akribeian compositus esse potero. Quod tunc te facile mihi condonaturum sperabo ... (?)

Sed ut ad rem ipsam veniam, magna voluptate orationem Excellentiae Tuae perlegi. Facunde, docte et pie lasseris sanguinis circulationem. Mire se torquere commentatores cuiusque generis in Ecclesiastae c. 12, v. 8, explicandi plusquam notum est. Quidam ea quod rebus extra corpus nostrum constitutis deferunt, alii corporis partibus accomando ludere potius quam veritatem elicare assere videntur. In tua Excellente Domine opinione Clarus Thomas Bartholinus aliique qui Epati funebria cecinerunt non admittent Epar fontem esse, dum ne guttula Chyli ad hepar feratur sed omnis ad cor per novum ductum chyliferum Pecqueti, quo Excellentiae Tuae notissimum non ambigo. Et fortasse ipsemet non amplius id defendis, cum jam decennium sit ex quo oratio haec exorata fuit. Claro Harvejo tunc haud displaceret, cui Venae lacteae non adeo probantur ut nuper ex Epistolis Clari Johanni Danieli Horstii p.m. 61 et seq. didici, quas tunc defendit Dominus Bartholinus in Epistola ad eundem Dominum Horstium annexa defensioni vasorum lacteorum et lymphaticorum contra Riolanum p. 170. Multis earundem lactearum existentiam et usum asseruit omni laude major Dominus Conring de Motu Chyli et sanguinis. Venasque notas et arterias hoc officio privavit c. 2 et 3. Caetera omnia tum in prosa quam inligata oratione suum pondus habent ac sufficiunt ad arguendum divini huius inventiosores.

Certe dolendum est Viros magnae apud studiosam juventutem authoritatis ut plurium ea respuere imo et insectari omnibus ingeniis quae ipsi non invenerunt, quamvis non solum pulchra sint sedet utilia imo scitu necessaria. Multi eo saepe vesaniae perveniunt ut et inquirere in rem inhonestum ducant, non quod laborem exhorrescant sed ne aliorum inventus assentire cogantur eum fugiunt. Dum apud Argentinenses literis operam dedi ab anno 39 ad 43, hospitio et mensa ac institutione Nobilis ac Celebris Domini Melchiori Sebizii gaudens nemini circulationem sanguinis innotuit. Ex Epistola Domini Walaei praegustum quendam habui, sed cum a praeceptoribus suc ...(?) haberi sentirem, eam haud magnifeci ceu non intellectam. Cum vero Mense Novembre 1643 Basileam pervenissem attentius Epistolam illam perlegi, ad experimenta obstupui, assensum tunc praebere non ausus fui. Veritas plane de novo medicum studium mihi occipiendum fore et si forte omnes scopulos superassem nemini Professorum Publicorum medicinam placituram, cum tum temporis paucissimi huius sententiae fautores exstiterint. Cum Claro Domino Platero in hac tum temporis periculum feci, re ad votum comperta penitus omnis vanus metus mihi excussus fuit magis magisque in sententiam illam abii. Sequenti anno Patavium profectus sum. Incidi tum in doctis- 
simos huius sententiae propugnatores Clarum Dominum Veslingium, Johannem Leonicenum, Dominum Thomam Bartholinum ex Silicia et Malta reducem, Dominum Gilianum Garz Hamburgensem Anatomicum accuratissimum et contubernalem meum, qui omnes quicquid dubii mihi superat quotidianis colloquiis et argumentis ex ipsa rei natura et oculari demonstratione penitus sustulerunt. Clarus Dominus Veslingius aurea suada in publicis lectionibus tantum non omnibus eam persuasit. Anno 1646 cum Romam pervenissem innotui ibi imprimis Domino Petro Servio viro doctissimo ac Nicolao Larche Lotharingo Chirurgo experientissimo ac Anatomico haud vulgari, at tum ipsis tum caeteris Romanis Medicis Circulatio Sanguinis peregrena erat. Domino Servio Conringium quem mecum habebam dono dedi. Perlegit equidem assensum nondum voluit praebere, pollicitus ulteriorem inquisitionem. Tunc temporis ad Clarum Marcum Aurelium Severinum Neapolim Roma scripsi, illuc profecturus nisi turbaetum ibidem gliscentes ac jam in flammam erumpentes obstitissent. Respondit mihi amice et prolixe multa esse quae sibi in hac probentur nondum tunc assentire cum Anastomoses arteriarum et venarum in habitu corporis tum ab ipso Harvejo negentur, tum in corpore non tot et tantae reperiuntur quae sufficerent ad concedendum toti massae sanguineae ex arteriis per venas reditum tam celerem ad cor.

Caeterum tum hoc tum reliquos scrupulos Clarus Marguardus Slegelius in doctissima commentatione de sanguinis motu contra Riolanum. Cum anno 1647 in Germaniam rediissem jam ubique a plurimis adprobari cognovi. Gratulor Germaniae nostrae magis magisque illam foveri et contra adversarios defendi. Imprimis id gaudendum est illam in plerisque Academiis strenuos et doctissimos propugnatores nactam fuisse vixque ulla est quae talibus destituatur. Clarus Rolfinkius Jenae, Mauritius Hoffmann Altorfii, Clarus Conradus Victor Schneider Wittenbergae, Clarus Conringius Helmstadii, Hafniae Bartholinus, Lovanii Plempius, apud Batavos plerique eam exosculantur.

(Randbemerkung) Utpote omnes Chartesinae philosophiae addictos qui (n) ullum (?) motum naturalem vectum esse admittunt omnem violentum egregie probant. Solar circulares naturales esse demonstrant ut ex ore Clari Domini St. Spleissii huius sententiae defensore ac arbitro felicissimo habeo. Qui Excellentiam Tuam plurimum salvare jussit. Cui et orationem tuum perlegere dono dedi.

Gratulor Tubingae vestrae Excell.T. obtigisse, nam nunc et in superioris Germaniae Academiis locum habitum auguror. Exulet adhuc Basilea, exulet Argentina, nihil tunc hoc veritati derogabit. Vivat et vigeat caeteris in locis imprimis autem Tubingae vestrae quae nobis post literas renatis maximos Medicos semper largita est. Erit ut posteri saltem Circulationem Sanguinis faveant. Senex ... (?) et veteribus opinionibus innutritos atque magistrorum sententiis juvamento obstrictos in ordinem redigi non possunt. Riolanus a quamplurimis vapulat. Imprimis a Bartholino supra notato. Eidem nuper admodum scripsi causa Nobilis cuiusdam viri nostratis sub finem Epistolae quaedam de sanguinis motu attexui, sed nullum dum responsum retuli. 
Ad observationem Excelltiae Tuae pervenio hac mihi pergrata erat. Nam in ea plurima annotatis digna animadverti et quae publicum lucem merentur. Singulari naturae providentia omnia imi ventris viscera adipe seu sevo quodam incrustata fuisse existimo, hinc tantum aquae vim ad triennium tolerare potuit. Epiglottidem eo quod cartilaginea sit non facile absumi opinor, quid si paralysi affecta fuisset et quod non raro hemiplegia aut apoplexia affectis contingit, unde deglutienda cum ad illum pervenerunt per os et nares evomunt. Aut forte eius membrana ab siccis salsis ab imo ascendentibus exhalationibus exsiccata et contracta fuisset ? Unde non recte clausa descendentibus imprimis potulentis aditum concessit, quae exquisitissimus interioris laryngis seu sus non admisit ac tanto impetu spiritus opem ferens ejaculabus (?) est. Cor sine pericardio numquam vidi, at cor pertinacissima ipsi coaluisse semel in vitrario quodam observavi. Corpora illa adiposa seu potius pituitosa in omnibus cachecticis inveni, imo plerisque fere qui ex morbo chronico diu decubui obierunt. Vidi ea saepe ovum columbinum superantia, radices profundas in utriusque ventriculis cordis anfractibus agentia ac ramos in venam cavam deorsumque in venam arteriosam ac arteriam venosam ut et aortam mittentia varieque pro ramorum ratione clivari cuta fuisse, imo ad epar et lienem pertigisse. Atque educta coralliorum instar ramosa fuisse. Cor omnibus vasis caeteris probe ligatis prope cavam inflatum in sinistro ventriculo intumuisse mirum est cum meminerim aliis hoc non successisse. Certum futurum argumentum aliquid e dextro in sinistrum transudare. Nisi forte foramen ovale e vena cava in arteriam venosam in faetu patulum in adulta hac praeter naturalis reseratum fuerit, ut nonnumquam accidere praeter naturalis Clarus Thomas Bartholinus et Clarus Veslingius concedunt. Botallus vero et Caecus Folius Anatomicus Venetii perpetuo hiare putarunt. Eiquidem praeter naturalis huic aegrae obtigisse suspicior dum ob corpora pituitosa venam arteriosam et arteriam venosam occupantia liber sanguinis transitus per pulmones ad sinistrum ventriculum negatus fuit, natura ut hanc mendam emendaret foramen hoc coacta reseravit, ut quantum posset individuum conservaret. Nuper admodum faeminam hydrope uterino et ascite enectam aperui. In utero ultra 16 mensuras in reliquo abdomine ultra 8 continebantur. Occasio hydropis uteri ulcus ad orificium internum uteri in dextro latere. Prolixe historiam Excellentiae Tuae transmitterem, sed obstat Tabellarii festinatio. Fasciculum observationum anatomicarum habeo quem luci crediturus sum, si vendibilem intellexero. Titulum quem his nundinis F. F. mittam Excellentiae Tuae cum disputatione mea de palpatione cordis transmitto, quod rogo boni consulas. Disputatio equidem immaturus faetus est. Multa insunt quae haud amplius defenderem. Sed manum de tabula. Vales his vir Clarus et Excellentus favore et amititia tua ulterius dignare prosum

Scaphusii, Cal. Sept. 1656

Excellento nomini Tuo

Saluta Dominum Lauterbachium addictissimus

et Dominum Simonium

J.J.W.MD. 
«Sei gegrüßt, Du vornehmer, ausgezeichneter und berühmter Herr, Gönner und Freund.

Schon lange war mir Deine vielseitige Bildung und besonders Deine, die Medizin erhellenden Studien, durch unseren gemeinsamen Freund, Herrn Simon ${ }^{12}$, bekannt. Schon lange wollte auch ich Dich um die Freundschaft angehen. Es ist ja bekannt, daß der in der Wissenschaft Aufgehende nicht leicht seinesgleichen entbehrt. Es waren nicht wenige, die es drängte, den in der Brust verborgenen Funken in eine helle Flamme zu entfachen. Hoffentlich kann ich Deinem Wunsche und Deinen Erwartungen genügen! Was mir der Briefwechsel wert ist, weiß ich sehr wohl. Und wenn ich zufällig dem einen oder andern nach dem Munde zu schreiben vermag, bin ich weit von den akademischen Übungen entfernt. Herr Simon weiß, wie ich durch die täglichen handwerklichen Verrichtungen von diesen Studien abgehalten werde. Im einen oder andern werde ich wohl zu Deiner Verfügung und zu akademischer Genauigkeit geeignet sein. Was Du mir sicher gewähren wirst, so hoffe ich ... (?)

Ich habe, um zur Sache zu kommen, mit großem Vergnügen Deine Rede gelesen ${ }^{19}$. Gewandt, gelehrt und richtig handelst Du den Blutkreislauf ab. Man weiß ja, wie merkwürdig sich die Kommentatoren jeglicher Art in den Ecclesiastae im Kapitel 12, Vers 8 winden: Gewisse bringen die Dinge, die sich außerhalb des Körpers befinden, vom Wege ab, andere scheinen eher mit angepaßten Teilen zu spielen, als die Wahrheit herauszulesen und zusammenzustellen. Nach Deiner Meinung lassen der berühmte Bartholin und die andern, die der Leber den Grabgesang gesungen haben, die Leber als Quelle nicht gelten, da kein Tröpfchen Chylus zur Leber geführt werde ${ }^{23}$, sondern alles zum Herzen durch den neuen Milchgang von Pecquet ${ }^{24}$. Es

23 Nachdem schon 1627 (posthum veröffentlicht) Gaspare Aselur (1581-1623) die Lymphgefäße und 1652 Jean Pecquet (1622-1674) den Ductus thoracicus am Hunde entdeckt und beschrieben hatten, fand Tномаs BARTholin ebenfalls 1652 den Ductus thoracicus am Menschen. Er beschrieb ihn in De lacteis thoracicis in homine brutisque nuperrime observatis historia anatomica, Kopenhagen 1652. Ein Jahr später erschien seine abschließende Monographie über die Lymphgefäße: Vasalymphatica nuper Hafniae in animalibus et homine inventa et hepatis exsequiae, Paris 1653. In dieser Schrift entthront er die Leber als zentrales, blutbildendes Organ im Sinne der hippokratisch-galenischen Lehre. Der lymphatische Abfluß erfolge über die Milchvenen in den Ductus thoracicus und erst dann ins Blut.

24 Jean Pecquet (1622-1674) studierte Medizin in Montpellier und schloß dort auch seine Studien ab. Er war ein Vertreter der Blutkreislauf-Lehre und setzte sich wegen Studienort und neuer Lehre in Gegensatz zu Jean Riolan fils, dem Dekan der Pariser Medizini- 
ist Dir bekannt, daß ich daran nicht zweifle. Vielleicht vertrittst Du Deine Rede nicht mehr, da sie schon vor zehn Jahren gehalten worden ist. Dem berühmten Harvey würde dies kaum mißfallen, dem die Milchvenen nicht so sehr genehm sind ${ }^{25}$, wie ich dies neulich aus den Briefen des berühmten Johann Daniel Horst, S. 61 ff., entnehmen konnte ${ }^{26}$. Diese Gefäße nimmt Herr Bartholin in einem Brief an denselben Herrn Horst in Schutz. Dieser Brief ist an die Verteidigung der Milch- und Lymphgefäße gegen Riolan, S. 170 angefügt ${ }^{27}$. Viel zu Existenz und Gebrauch dieser Milchvenen hat Herr Conring in seinem Buch über die Bewegung des Chylus und des Blutes zu aller größter Zufriedenheit beigefügt. Die schon bekannten Venen und Arterien beraubt er dieser Eigenschaft (= Resorption, Verfasser) im Kapitel 2 und $3^{28}$. Alles übrige in Prosa und Versen hat sein Gewicht und genügt für den Beweis des Göttlichen all dieser Entdecker.

Man muß aber hervorheben, daß Männer, die bei der eifrigen Jugend ein großes Ansehen besitzen, am meisten das verachten und sogar jedes Talent verfolgen, was sie selbst nicht gefunden haben, obwohl es nicht nur vortrefflich, sondern auch nützlich, ja sogar nötig ist. Viele verfielen dem Wahnsinn und stöberten sogar unehrenhafte Dinge auf. Nicht weil sie die Mühe scheuen, fliehen sie die fremden Entdeckungen, sondern damit sie nicht ge-

schen Fakultät. Daraus entstanden verschiedene Streitschriften. 1651 beschrieb Pecquet als Erster das Receptaculum (cysterna) chyli in Experimenta nova anatomica, quibus incognitum hactenus chyli receptaculum, et ab eo per thoracem in ramos usque subclavios vasa lactea deteguntur, Paris 1651.

25 William Harvey (1578-1657) war keineswegs eingenommen für Bartholins Ansicht der lymphatischen Resorption der Nahrung. Diese erschien ihm unverständlich, da doch, auch vergleichend anatomisch, sehr viele Venen vorhanden seien, die nach Bartholins Ansicht keine Funktion haben sollten. Harveys Ansicht der venösen Nahrungsresorption sollte erst im 19. Jahrhundert bewiesen werden.

26 W. HARVEY, Exercitationes duae anatomicae de circulatione sanguinis ad Joannum Riolanum filium, Roterodami 1649.

27 Th. Bartholin, Defensio vasorum lactearum et lymphaticarum adversus Joannem Riolanum, Hafniae 1655.

${ }^{28}$ Hermann Conring (1606-1681) war ein Vielschreiber und hat neben medizinischen Schriften eine ganze Reihe bedeutender politischer Schriften zu Problemen seiner Zeit veröffentlicht. Er studierte in Leiden und Helmstedt Medizin und Philosophie. Er ist in seinen medizinischen Schriften ein Verfechter der lymphatischen Resorption. Auch Wepfer bekennt sich zur lymphatischen Resorption der Nahrung und steht somit auf der Seite von Bartholin, Conring usw. Wepfer hat sich auch schon in der Studienzeit für dieses Problem interessiert und darüber unter von Brunn in Basel disputiert. (J. J. WEPFER, Disputatio de Chylificatione, Basileae 1644). 
zwungen werden ihnen zuzustimmen. Als ich in den Jahren 39 bis 43 bei den Straßburgern studierte, die Tafel, das Haus und die Vorlesungen des vornehmen und erhabenen Herrn Melchior Sebiz genießend ${ }^{29}$, war niemandem der Blutkreislauf bekannt. Aus den Briefen des Herrn de Wale ${ }^{30}$ bekam ich einen Vorgeschmack, aber als ich bemerkte, daß die Lehrer mir zu folgen begannen, spielte ich diese Erkenntnis nicht hoch, wie wenn ich sie nicht verstanden hätte. Als ich aber im November 1643 nach Basel kam, las ich diesen Brief aufmerksamer durch, war erstaunt über die Versuche, wagte aber noch nicht einen Nachhall zu versuchen. Denn ich hätte das medizinische Studium nochmals beginnen müssen, und wenn ich vielleicht auch alle Klippen überwunden hätte, wäre diese Medizin doch keinem der Professoren genehm gewesen, da in jener Zeit noch sehr wenige Befürworter für diese Ansicht eintraten. Mit dem berühmten Platter ${ }^{31}$ machte ich in jener Zeit Versuche. Was ich aber erfuhr, beseitigte fast jeden leeren Zweifel, und mehr und mehr ging ich zu dieser Ansicht über. Im folgenden Jahre reiste ich nach Padua. Ich fiel sozusagen auf die gelehrtesten Verfechter dieser Ansicht: den berühmten Wesling ${ }^{32}$, Johann Leonicenus, Herrn Thomas Bartholin ${ }^{18}$, soeben aus Sizilien und Malta zurück, Herrn Gilian Gartz, einen sehr sorgfältigen Anatomen aus Hamburg ${ }^{33}$, der mein steter Begleiter war. Sie alle zerstreuten fast alles, was immer an Zweifeln übrigblieb im täglichen Gespräch, mit Beweisen aus der Natur selbst und augenscheinlichen

29 Melchior Sebiz II (1578-1674) soll an 27 Universitäten studiert haben. Er promovierte zum Doktor der Medizin in Basel 1610. Seine Professur der Medizin erhielt er 1621 an der Universität Straßburg. Damit war er auch ein Lehrer Wepfers, als dieser in den Jahren 1639-1643 dort studierte. 1625 wurde Sebiz Stadtphysukus von Straßburg und 1630 wurde er von Ferdinand II. zum Pfalzgrafen ernannt. Er hat sehr viel geschrieben und wurde durch die Herausgabe des Kräuterbuches von Tragus bekannt (4. Ausgabe, Straßburg 1630).

30 JAN DE WALE (1604-1649), Epistolae duae de motu chyli et sanguinis ad Thomam Bartholinum, Casparis filium, o. O. 1640. Später in Th.BARTholin, Institutiones anatomicae, Lugdunum Batavae 1645.

31 Felix Platter II (1605-1671) wurde am 1. August 1605 zu Basel geboren. Er promovierte 1624 zum Doktor der Philosophie und 1629 zum Doktor der Medizin. Bereits 1630 wurde er zum Professor der Logik in Basel ernannt und 1633 zum Professor der Physik. 1651 wurde er Stadtphysikus und 1664 Senator. Er starb am 3. Juni 1671.

32 Johann Wesling (1598-1649) aus Minden, in Westfalen gebürtig, wurde 1632 Professor für Anatomie und Chirurgie in Padua und war einer der Lehrer Wepfers.

33 Gilian Gartz war Arzt und Anatom in Hamburg. Er wird auf dem Titelblatt der Streitschrift von Paul Marquard Schlegel gegen Jean Riolan fils als zweiter Anatom erwähnt (Gesnerus 21, [1964] 74). 
Darlegungen. Der berühmte Herr Wesling überzeugte in den öffentlichen Vorlesungen mit seiner Überredungskunst nicht alle.

Als ich 1646 nach Rom ging, lernte ich dort vor allem Herrn Pietro Servi ${ }^{34}$, einen sehr gelehrten Mann, und Nicolaus Larche ${ }^{35}$, einen sehr erfahrenen Chirurgen und außergewöhnlichen Anatomen aus Lothringen, kennen. Aber damals war diesen und den übrigen römischen Ärzten der Blutkreislauf fremd. Herrn Servi schenkte ich den Conring ${ }^{36}$, den ich bei mit hatte. Er las ihn, allerdings wollte er seine Zustimmung noch nicht geben, versprach aber weitere Erforschung ${ }^{37}$. Damals schrieb ich dem berühmten Marc Aurel Severin $^{38}$ nach Neapel, ich würde dorthin reisen, wenn die schon ausgebrochenen und zunehmenden Unruhen es erlauben würden. Er antwortete ${ }^{39}$ freundschaftlich, es sei sehr vieles, was ihm glaublich erscheine, aber er könne noch nicht zustimmen, da die Verbindung der Arterien und Venen im Körper eben auch von Harvey verneint werde, da im Körper auch nicht so viele Gefäße gefunden würden, die genügten, um einen raschen Rückfluß einer solchen Blutmenge aus den Arterien durch die Venen zu gewährleisten. Im übrigen enthob mich besonders dieser und der übrigen Bedenken der berühmte Paul Marquard Schlegel in seinem gelehrten Kommentar über den Blutkreislauf gegen Riolan ${ }^{40}$. Als ich 1647 nach Deutschland zurück-

34 Pietro Servi (gestorben 1648) war in Rom Professor der Medizin und in vielem ein Anhänger Galens. Er versuchte eine Synthese von Galens Lehre mit derjenigen von Paracelsus. Georg Christoph Schelhammer, der 1687 Hermann Conrings Introductio in totam medicinam herausgab, beurteilt Servi in den Anmerkungen wie folgt: «Zur selben Zeit lebte dort (in Rom) Pietro Servi, ebenfalls sehr gelehrt, der verschiedenes schrieb. In einigem schien er der Galenischen Doktrin zu folgen, obwohl er auch davon abgeht, wie im Buch über die Salben.»

35 Nicolaus LARChE wird in WePFERS Observationes ... de affectibus capitis nochmals erwähnt. Andere Angaben über ihn konnten bisher noch nicht gefunden werden.

36 Wahrscheinlich ist es Hermann Conring, Dissertatio de sanguinis generatione et motu naturali, Lugdunum Batavae 1643.

${ }^{37}$ Dieser eher zurückhaltenden Stellungnahme zum Blutkreislauf entspricht auch der Wertung durch G. Ch. Schelhammer. Vgl. Anm. 34.

38 Marc Aurel Severin (1580-1656) studierte Philosophie und Medizin in Neapel und promovierte zum Doktor der Medizin 1606 in Salerno. 1610 wurde er Professor für Anatomie und Chirurgie in Neapel.

${ }^{39}$ Ein solcher Brief Severins an Wepfer konnte in keinem der Wepfer-Nachlässe (Zürich und Leiden) bis jetzt gefunden werden.

40 Paul Marquard Schlegel (1605-1653) war Physikus der Stadt Hamburg seit 1642 und schrieb De sanguinis motu commentatio, in qua praecipue in Joh. Riolani, V.C. sententiam inquiritur, Hamburg 1650. 
kehrte $^{41}$, bemerkte ich, daß bereits überall die meisten zustimmten. Ich bebeglückwünsche unser Deutschland, daß es diesen immer mehr fördert und gegen die Gegner verteidigt. Es ist erfreulich, daß an den meisten Akademien unternehmende und sehr gelehrte Verfechter sich erhoben haben, und es ist kaum noch eine, die von ihnen im Stiche gelassen wird, der berühmte Rolfink in Jena ${ }^{42}$, Mauritius Hofmann in Altorf ${ }^{43}$, der berühmte Conrad Victor Schneider in Wittenberg ${ }^{44}$, der berühmte Conring in Helmstedt ${ }^{28}$, in Kopenhagen Thomas Bartholin ${ }^{18}$, in Löwen Plempius ${ }^{45}$ und bei den Holländern bekennen sich die meisten dazu.

(Randbemerkung.) Alle nämlich, die sich der Cartesianischen Philosophie verschrieben haben, beweisen besonders heftig, daß viele Bewegungen ein natürlicher Vorgang seien. Sie zeigen, daß der Sonnenkreis natürlich ist, was ich aus dem Munde des berühmten Herrn Stephan Spleiß $\beta^{46}$ hörte, eines Verteidigers und glücklichen Beurteilers dieser Lehre. Er läßt Dich herzlich grüßen; auch ihm habe ich Deine Rede zum Lesen gegeben.

41 J. J. Wepfer promovierte am 31. Juli 1647 in Basel zum Doktor der Medizin. Es ist bemerkenswert, daß er das Empfinden hat zu Deutschland zu gehören. Später zeigte sich dann, daß sich seine Praxis vor allem im süddeutschen Raum ausdehnen sollte.

42 Werner Rolfinck (1599-1673) promovierte 1625 in Padua und wurde 1629 Professor für Anatomie, Chirurgie und Botanik in Jena. Bei den Laien stand er im Rufe Leichen zu stehlen, und die Verbrecher sollen sich damals ausbedungen haben, nach ihrer Hinrichtung nicht «gerolfinckt» (= seziert) zu werden.

43 Mauritius Hofmann (1622-1698) promovierte 1645 in Altorf und wurde dort 1648 Professor für Anatomie und Chirurgie. Er ist der eigentliche Entdecker des Ductus pancreaticus (164l am Truthahn), deutete ihn jedoch fälschlicherweise als Chylusgefäß. Johann Georg Wirsung (1600-1643) beschrieb ihn dann 1642 am Menschen.

44 Conrad Victor Schneider (1614-1680) promovierte 1639 zum Doktor der Medizin und wurde später in einem gewissen Sinne Wepfers Rivale. Schneider veröffentlichte 1660 in Wittenberg sein Werk De catarrhis, in welchem er sich gegen die allgemeine Ansicht, daß der Nasenschleim aus dem Gehirn ausfließe, aussprach, da dies anatomisch unmöglich sei. Wepfer hatte diese Ansicht bereits 1658 in seinem Buch über die Apoplexie, S. 140 bis 142, erwähnt. Zu Streitigkeiten ist es aber zwischen den beiden nicht gekommen.

45 Vopiscus Fortunatus Plempius (gestorben 1671) wurde 1633 Professor der Medizin in Löwen. Er nahm 1644 öffentlich für den Blutkreislauf Harveys Stellung. Auf der anderen Seite war er ein Gegner der China-Rinde und verhinderte deren Einführung in den Niederlanden mit seiner Schrift Antinus Conringius Peruviani pulveris defensor, repulsus a Melippo Protymo, Löwen 1655. Melippo Protymo ist das Pseudonym von Plempius.

46 Stephan Spleiss (1623 [15 ?]-1693) war Mathematiker, Optiker und Astronom. 1649 erwarb er sich das Bürgerrecht der Stadt Schaffhausen, wurde 1652 Konrektor des Gymnasiums und 1655 Rektor. 1671 wurde er Professor für Logik und Mathematik. Nach Wepfers Aussagen war er ein Anhänger von Descartes. 
Ich beglückwünsche Euer Tübingen, daß Du dort bist, denn ich ahne, daß Dir auch unter den höheren Akademien Deutschlands ein Platz zufallen wird. Verwaist ist Basel, ebenso Straßburg, nichts wird sich dieser Wahrheit entziehen können. Die Lehre vom Blutkreislauf möge aufleben und gedeihen an den übrigen Orten, besonders aber in Eurem Tübingen, das uns gegenüber, den Studien Entwachsenen, meistens Ärzten, immer freigebig war. Wenigstens werden unsere Nachfahren den Blutkreislauf begünstigen. Die Greise aber und die, die mit alten Ansichten gefüttert worden sind und auch in Lehrsätzen von Meistern verstrickt sind, können nicht in diese Reihe aufgenommen werden. Riolan wird von allen möglichen durchgehechelt. Besonders von Bartholin in der oben erwähnten Schrift. Ihm habe ich kürzlich wegen eines vornehmen Herren geschrieben. Am Schluß des Briefes habe ich einiges über den Blutkreislauf angefügt, aber noch keine Antwort erhalten ${ }^{47}$.

Ich komme nun zu Deiner Beobachtung. Sie war mir sehr willkommen, denn ich bemerkte in ihr mehreres, was einer Anmerkung würdig ist und veröffentlicht zu werden verdient. Ich glaube, daß durch die besondere Voraussicht der Natur alle Eingeweide des Unterbauches mit Fett oder Talg belegt wurden, weshalb ein solcher Wasserdruck bis zu drei Jahren ausgehalten werden konnte. Ich glaube nicht, daß die Epiglottis leicht aufgelöst werden kann, gerade weil sie knorpelig ist. Wenn sie gelähmt gewesen wäre, was nicht selten bei Kranken mit Hemiplegie und Apoplexie geschieht, wäre das Geschluckte, das dorthin gelangt, durch Mund und Nase erbrochen worden. Oder vielleicht ist deren Membran von trockenen, salzigen oder sogar aufsteigendenden Ausdünstungen ausgetrocknet und zusammengezogen worden? Davon würde dann bei schlechtem Schluß, besonders bei Flüssigem, etwas davon eindringen. Dies läßt jedoch der untere Kehlkopf oder auch weiter oben aufs sorgfältigste nicht zu, und unter Zuhilfenahme eines Atemstoßes wirft er es aus. Ein Herz ohne Herzbeutel habe ich nie gesehen, aber einmal habe ich bei einem Glasbläser eine starke Verwachsung beider beobachtet. Jene fettigen oder vielmehr schleimigen Körper habe ich bei allen Ausgezehrten gefunden, sogar bei solchen, die nach langem Krankenlager an einer chronischen Krankheit starben. Ich sah, daß sie öfters größer als ein Taubenei waren, ihre Wurzeln tief in die Winkel beider Herzkammern senkten und ihre Zweige nach oben in die «Vena arteriosa » (Lungen-

47 Ein Brief Bartholins an Wepfer konnte noch nicht gefunden werden, weder in Zürich noch in Leiden. 
arterie) und «Arteria venosa» (Lungenvene) wie auch in die Aorta schickten und in einzelne Äste verzweigt waren. Sie gelangen sogar bis zur Leber und Milz. Nimmt man sie heraus, sehen sie wie Korallenzweige aus. Es ist merkwürdig, daß sich die linke Kammer bläht, wenn man alle Gefäße gut zubindet und das Herz von der Hohlvene her aufbläst. Wie ich mich erinnere, passierte dies andern nicht. Sicher wird man behaupten, etwas von der Luft werde von rechts nach links befördert (transudare), außer natürlich wenn zufällig das ovale Fenster aus der Hohlvene zur «Arteria venosa» offenstände, was beim Foetus natürlich, beim Erwachsenen jedoch widernatürlich ist. Der berühmte Thomas Bartholin ${ }^{18}$ und Wesling ${ }^{28}$ haben zugegeben, daß es manchmal widernatürlicherweise zutrifft, Botallo ${ }^{48}$ aber und der Venezianer Anatome Caesus Folli ${ }^{49}$ glauben, es stehe ständig offen. Bei dieser Krankheit glaube ich jedoch, daß es widernatürlich war, weil durch die Schleimkörper, die die Lungenarterie und Lungenvene verlegten, der freie Durchgang des Blutes durch die Lungen zur linken Herzkammer verhindert war. Die Natur, so gezwungen diesen Fehler zu beheben, hat das Foramen geöffnet, um das Individuum nach Möglichkeit zu retten. Neulich habe ich eine Frau, die an «hydrops uteri» und Bauchwassersucht starb, geöffnet. Im Uterus waren über $16 \mathrm{Ma}$ (=16,6 Liter!), im übrigen Bauch über $8 \mathrm{Maß}$ Flüssigkeit zu finden. Der Grund des Hydrops uteri war ein Geschwür am innern Muttermund auf der rechten Seite. Gerne würde ich Dir die Geschichte schicken, aber der Postabgang (festinatio Tabellarii) hindert dies. Ich habe einen Faszikel anatomischer Beobachtungen, die ich zu veröffentlichen gedenke, wenn sich der Verkauf lohnt ${ }^{50}$.

Die Schrift, die ich Dir diesmal sende, übermittle ich Dir zusammen mit meiner Disputation über das Herzklopfen ${ }^{51}$. Magst Du sie gutheißen. Die

48 Leonardo Botallo (geboren um 1530) war Arzt von Asti im Piemont. Er promovierte in Pavia und wurde 1575 Leibarzt von Heinrich III. in Frankreich. Er befürwortete einen ausgedehnten Aderla $\beta$ und führte ihn in Frankreich ein.

49 Caecus Folli (1615-um 1650) hatte in Venedig den Lehrstuhl für Anatomie inne und schrieb einen Tractatus de via sanguinis a dextro in sinistrum cordis ventriculum defluentis, Venedig 1639.

50 J.J. WEPFER veröffentlichte diese anatomischen Beobachtungen unter dem Titel $O b$ servationes anatomicae ex cadaveribus eorum, quos sustulit apoplexia im Jahre 1658 in Schaff hausen. Eine zweite Ausgabe, zum Teil erweitert mit Beobachtungen aus Johann Burgauers II. (gestorben 1635 an der Pest), des Stadtarztes von Schaffhausen, Praxisbüchern.

51 J.J. WePFER, Dissertatio inauguralis de palpatione cordis, Basileae 1647. 
Disputation ist ein unreifer Foet, worin vieles steht, das ich nicht weiterhin vertreten möchte. Aber genug.

Lebe wohl, berühmter und vornehmer Herr, und bedenke mich weiterhin mit Deiner Gunst und Freundschaft.

Schaffhausen, 1. September 1656

Grüße Herrn Lauterbach ${ }^{52}$ und Herrn Simon ${ }^{12}$.»

Dein Dir sehr ergebener J.J.Wepfer

Wepfer entschuldigt sich zunächst in einem etwas gewundenen Stil, der nicht zu seiner sonst klaren und eindeutigen Art paßt. Seine Prägnanz bemerkt man schon auf den folgenden Seiten, wo er das Thema anpackt. Es zeigt sich, daß er über die Veröffentlichungen, die für oder gegen Blutkreislauf und Lymphgefäßsystem geschrieben wurden, genau orientiert ist. Auch wertet er sie mit eigenen und klaren Gedanken. Wepfer berichtet im weiteren wie er zur Überzeugung kam, daß der Blutkreislauf richtig und wahr sei und gibt dabei genaue autobiographische Angaben. Nach seiner autobiographischen Darstellung, die eng mit den damaligen medizinischen Problemen verflochten dargestellt wird, erörtert er noch Brotbecks Beobachtungen, die ihm jener als Manuskript zugestellt hatte. Er stellt einen anatomischen Irrtum richtig, so das permanente Offenstehen des Foramen ovale im Herzen.

Sehr bemerkenswert ist ganz am Ende des Briefes der Satz über die anatomischen Beobachtungen, die Wepfer zu veröffentlichen gedenkt, «si vendibilem intellexero ».

Zwei Jahre später erst antwortete Brotbeck auf diesen Brief Wepfers. Wepfer hatte unterdessen seine anatomischen Beobachtungen unter dem Titel Observationes anatomicae ex cadaveribus eorum quos sustulit apoplexia 1658 in Schaffhausen herausgegeben. Brotbeck schreibt Ende 1658 an Wepfer ${ }^{53}$ :

Nobilis, Excellentissime et Experientissime Vir, Domine Fautor ac Amice percolende.

Serius justo, meum parturire, elephantum more, calamum, jure dixeris, ei quod ad biennale ferme intervallum, meae ad lectissimas tuas, lectu videlicet dignissimas, responsoriae sufflamen plurimum passae fuerint. Nunc vero cum anni huius

52 Wolfgang Adam Lauterbach (1616-1678) war Professor der Jurisprudenz in Tübingen seit 1648 und Württembergischer Geheimrat.

${ }^{53}$ ZBZ, Ms Z VII 295.7, S. 5-6 (Text) und S. 8 (Anschrift). 
exodio, literas rursus exordium faciam, aurem atque pennam, mihi vellicante, nostro Domino Simonio, affine amicoque meo jucundissimo, qui se Scaphusium iter meditari, nuper mihi denunciavit. Ad respondendum vero, praeter benevolum Tuum in me affectum, tua insuper me invitat eruditio, Medicumque forum ampliandi atque locupletandi industria. Illam abunde testatum reddidere, doctissima tua, juxtaque solidissima de sanguinis circensibus argumenta, quae tuarum, ad me literarum condimentum mihi gratissimum fuere. De cuius dogmatis veritate, tam certus nunc sum, quam certissimus, sive enim rationes, sive rationum testes, sive utriusque partis sensualem fidem requisivero, neutrubique ...(?) tua, evidenter admodum, ex observationibus tuis anatomicis innotescit, quarum promulside jampridem, ita me inescasti, ut totum nunc alvearium inhiem, nullus equidem ambigo, primum illud mellificium tuum anatomico-medicum et calamo et prelo otia jam dudum ferisse. Idem etiam epigrammate aliquo si non digno satis, debito tamen condecorare, et per fratrem tuum Argentinae tum commorantem, transmittere tibi non inconcinnum, nec ingratum fore duxi. Multam eiquidem hac in palaestra laudem commereris, quia non multorum, sed paucorum in arte nostra virorum vestigia premis, Tulpii puto, Th. Bartholini, Spigelii, Fabritii Hildani, qui non vanum hoc extispicii genus insigniter prae ceteris excoluere, mirisque modis aetiologiam et pathologiam illustrarunt. Nec mihi unquam atque uspiam in re dubita atque obscura, major, quam ex hoc ipso speculo, lux affulgere et potuit et poterit. Insistas igitur, quaeso, faveatque his lauris, ausisque tuis Deos! Haecce levia et brevia jam boni consule, nox enim ferme media, medium scribendi cursum abrumpit, at coronidis loco, felicissimum anni huius excursum, novique reducis decursum desuper tibi apprecatur. Vale, Medicorum ocelle, meque, ut jam concepisti, amare literisque tuis affari non desine.

Cursim

Tubingae e Museo

...(?) Emmanuelis nostri natalitiis 1658
Excellentiae Tuae

Addictissimus

Jo. Conradus Brotbekius

«Vornehmer, sehr berühmter und erfahrener Mann, sehr geehrter Herr, Gönner und Freund.

Meinen Brief trage ich solcherart bis fast zu zwei Jahren in mir herum, daß Du mit Recht sagst, wie ein Elefant ${ }^{54}$. Meine Antwort auf Deinen sehr gediegenen und lesenswerten Brief mußte sich einen Anstoß recht spät gefallen lassen. Nun aber mit dem Ausgang des Jahres beginne ich wieder mit den Briefen. Ohr und Feder mahnen mich in der Person unseres Herrn Si-

54 Die Tragzeit der Elefanten beträgt 20-25 Monate und scheint demnach Brotbeck und den damaligen Gelehrten geläufig gewesen zu sein. 
mon $^{12}$, meines Schwagers und sehr lieben Freundes, der sich für eine Reise nach Schaffhausen vorbereitet, wie er mir neulich ankündigte. Neben Deiner mir wohlwollenden Zuneigung fordert mich auch noch Deine Bildung heraus, eine Antwort zu schreiben; ebenso Dein Fleiß, der das ärztliche Leben erweitert und bereichert. Diesen bezeugen auch Deine sehr gelehrten und auch fundierten Beweise über die Kreisläufe (circenses) des Blutes hinlänglich, die Deinen Briefen eine sehr willkommene Würze gaben. Über die Wahrhaftigkeit dieser Lehre bin ich nun völlig überzeugt; wenn ich nach den Kenntnissen oder den Zeugen dieser Kenntnisse oder nach sinnlicher Wahrheit beider Teile forschte, fehlte mir der Weg keineswegs. Dein bisheriger Fleiß, ganz offenbar, ist aus Deinen anatomischen Beobachtungen bekannt, mit denen Du schon vorher mich so geködert hast, daß ich nun nach dem ganzen Bienenkorb verlange. Ich zögere keineswegs Deine Honigwabe mit dem Rohr zu schlagen, und in der Muße bearbeite ich sie schon längst mit der Kelter. Ich habe mit einem, wenn auch nicht würdigen, so doch gebührenden Epigramm ${ }^{55}$ Dich zu ehren gesucht, und ich lasse es Dir durch Deinen jetzt in Straßburg weilenden Bruder ${ }^{56}$ zukommen. Ich hoffe, es sei Dir nicht ungereimt und ungelegen. Hier an der Schule verdienst Du großes Lob, weil Du nicht den Spuren vieler, sondern weniger Männer unserer Kunst folgst, so Tulpius ${ }^{57}$, Thomas Bartholin ${ }^{18}$, Spiegel ${ }^{58}$, Fabritius Hildanus ${ }^{59}$, die vor allem nicht diese erfolglose Art von Eingeweideguckern ausbilden und Ätiologie und Pathologie erstaunlich erhellen. Niemals und nirgendwo konnte in einer unklaren und dunklen Angelegenheit das Licht stärker aufblitzen als in diesem Spiegel, und es wird es noch können. Bleibe dabei, ich bitte Dich, und Gott möge Dich mit seiner Gunst und Dein Unternehmen segnen. Heiße diesen unbedeutenden und kurzen Brief gut. Es ist fast Mitternacht, die Mitte unterbricht den Lauf des Schreibens. Anstelle des Schlußschnörkels wünsche ich ein glückliches Jahresende und ein gutes

55 Dieses Epigramm hat sich vorläufig noch nicht gefunden.

56 Johannes Wepfer (1635-1670), der jüngere Bruder Johann Jakobs, studierte ebenfalls Medizin. Er starb mit 35 Jahren an einer «Pleuritis saevissima».

57 Niclaus Tulp (1593-1674) studierte in Leiden Medizin und wurde daselbst Schöffe und Bürgermeister.

58 Adrian Spiegel (1578-1625) studierte in Löwen und Padua Medizin. Er praktizierte dann in Mähren und wurde 1615 als Professor für Anatomie und Chirurgie nach Padua berufen.

59 Wilhelm Fabry von HiLden (1560-1634) stammt aus Deutschland und war seit 1615 bis zu seinem Tode ein berïhmter und geachteter Chirurg und Stadtwundarzt von Bern. 
neues Jahr. Leb wohl, Du Augapfel der Ärzte, bleibe mir weiterhin verbunden und hör nicht auf Dich der Wissenschaft zu widmen.

In Eile, Tübingen, Bibliothek

Weihnachten 1658
Dein Dir sehr ergebener

Johann Conrad Brotbeck.»

Dieser zweite Brief Brotbecks spiegelt seine Bewunderung für Wepfer wider und ist im Tone auch wesentlich weniger maniriert und freundschaftlicher. Am meisten scheint Brotbeck von Wepfers Fleiß beeindruckt zu sein. Im ganzen ist jedoch auch hier der Stil schwülstig und arm an Substanz. Für das Biographische in Wepfers Leben erfahren wir, daß sein Bruder Johannes in diesem Jahr (1658) in Straßburg studiert.

Damit scheint der soeben begonnene Briefwechsel bereits unterbrochen. Die fünf Briefe Brotbecks an Wepfer aus den Jahren 1673-1775 konnten hier nicht berücksichtigt werden. Briefe oder Briefkopien Wepfers aus dieser Zwischenzeit sind bis jetzt noch nicht bekannt, ebensowenig solche von Brotbeck. Möglicherweise sind noch solche beider Ärzte im vorläufig noch verschollenen Brotbeck-Nachlaß zu finden.

\section{Zusammenfassung und Wertung}

Aus diesem Briefwechsel Brotbeck -Wepfer erfahren wir über Brotbeck, der den Briefwechsel begann, daß er Stadtarzt in Tübingen war und sich schon mit seiner Inauguraldissertation für den Blutkreislauf eingesetzt hatte. Er selbst sucht im Briefwechsel Anregung für Sektionen und Sektionsbefunde bei Wepfer. Zu diesem Zweck überläßt er ihm ein Manuskript zur Beurteilung, erbittet es sich aber wieder zurück. Der Vermittler zwischen den beiden Ärzten ist der spätere Leibchirurg des Fürstenbergischen Geschlechts in Donaueschingen, Johann Christoph Simon. Brotbeck tönt in seinem ersten Brief die Streitschriften zwischen Jean Riolan, dem Dekan der Pariser Fakultät, und dem dänischen Anatomen Thomas Bartholin an. In seinem zweiten Brief an Wepfer ergeht sich Brotbeck in Lobeserhebungen über dessen Fleiß im Buch über den Hirnschlag. Wir erfahren, daß sich Wepfers Bruder Johannes zum Studium in Straßburg aufhält.

Brotbecks Erwähnung seiner Dissertation über den Blutkreislauf und der Streitschriften zwischen Riolan und Bartholin muß Wepfer zu einer Stellungnahme herausgefordert haben. Wepfer berichtet ausführlich wie er zur Lehre des Blutkreislaufes gekommen ist. Der Anstoß sich damit zu be- 
schäftigen waren zwei 1641 von Daniel Horst veröffentlichte Briefe zu diesem Thema. Aber in Straßburg, wo Wepfer 1639 bis 1643 studierte, wobei er bei Melchior Sebiz II wohnte und dessen Vorlesungen besuchte, wagte er noch nicht die neue Lehre vor den Professoren zu erwähnen. Aber auch in Basel, wohin er im November 1643 wechselte, kommt es noch nicht zu einem öffentlichen Bekenntnis, wohl aber zu eingehenden Studien der Briefe und Versuchen zusammen mit Felix Platter II. Wepfer wechselt ein Jahr später wiederum die Universität und reist nach Padua. Damit war er sozusagen an der Quelle angelangt. Nach seinen eigenen Aussagen muß damals Padua die Hochburg der Verfechter des Kreislaufes gewesen sein. Seine Lehrer in dieser Beziehung waren Vesling, Bartholin, Gartz und LeoNICENUS, über den bisher leider nichts in Erfahrung gebracht werden konnte. Wahrscheinlich lernte Wepfer Thomas Bartholin persönlich kennen, war doch dieser seit 1642 «Protektor der Deutschen Nation» in Padua. 1645 finden wir Wepfer in Venedig, wobei es sich allerdings auch nur um einen Abstecher von Padua aus gehandelt haben kann. Diese Angabe Wepfers wurde einem anderen Briefe entnommen, und er gibt auch nicht an, wie lange er sich in Venedig aufgehalten hat. 1646 reiste er nach Rom und wäre auch nach Neapel zu Severin gereist, wenn dort nicht Unruhen ausgebrochen wären. Die Römer Ärzte und auch Severin nahmen die neue Kreislauflehre nur bedingt an.

Da Wepfer keine Abenteurernatur war, wagte er die gefährliche Reise nach Neapel nicht und kehrte 1647 in die Schweiz zurück. Er schloß sein Studium am 31.Juli 1647 in Basel mit der Dissertation Über das Herzklopfen und seiner Promotionsrede Über Trinkkuren mit Thermalwässern ab. Bisher war nur bekannt gewesen, daß Wepfer an den drei Universitäten Straßburg, Basel und Padua insgesamt zehn Jahre Medizin studiert hatte. Es waren jedoch darüber keine so eingehende Einzelheiten zu finden gewesen.

In seinem Brief wird Wepfer auch recht ausfällig gegen solche, die sich abwehrend zum Kreislauf einstellen, so z.B. die Sätze: «Nicht weil sie die Mühe scheuen, fliehen sie die fremden Entdeckungen, sondern damit sie nicht gezwungen werden ihnen zuzustimmen» und "Wenigstens werden unsere Nachfahren den Blutkreislauf begünstigen. Die Greise aber und die, die mit alten Ansichten gefüttert worden sind und auch in Lehrsätzen von Meistern verstrickt sind, können nicht in diese Reihe aufgenommen werden.»

Im weiteren erfahren wir aus Wepfers Brief Einzelheiten über die damaligen Streitschriften um den Blutkreislauf, wobei er sich sehr belesen zeigt. Auch sein Kommentar zu Brotbecks Manuskript zeugt von seiner Belesen- 
heit und scharfen Beobachtungsgabe. So weiß er bereits, daß das Verschlukken beim Apoplektiker auf schlechten Schluß der Epiglottis zurückzuführen ist. Allerdings ist dann die Erklärung dafür reichlich barock und unklar. Es ist ihm auch bekannt, daß das Foramen ovale nur beim Foeten offensteht und beim Erwachsenen geschlossen zu sein hat. Die Bedeutung des kleinen Kreislaufes ist ihm, wie übrigens hundert Jahre später auch dem großen Physiologen Albrecht von Haller, noch nicht bekannt.

Sehr aufschlußreich ist die Bemerkung Wepfers, daß er ein Buch anatomischer Beobachtungen herausgeben werde, wenn der Verkauf sich lohne. Er scheint mit Geldmitteln nicht überreich gesegnet gewesen zu sein.

Wenn wir eine abschließende und zusammenfassende Wertung dieses Briefwechsels zu geben versuchen, so ist zweifellos Wepfer der bedeutendere dieser beiden Männer, mit klaren Ideen und scharfer Beobachtungsgabe und gleichsam prädestiniert einmal der führende Kopf der freien Schaffhauser Ärzteschule zu werden. 